\title{
The Optimized Design of the Key Components of Mechanical Rotary Stereo Garage Based on ANSYS
}

\author{
Shi-Bin $\mathrm{CHEN}^{1,2, a,}$ Min-Dong ZHANG ${ }^{1,2, b}, \mathrm{Na} \mathrm{Yu}^{1,2, \mathrm{c}}$, Guan-Long SUN ${ }^{1,2}$ and \\ Wei-She ZHANG ${ }^{1,2}$ \\ ${ }^{1}$ National Engineering Laboratory for Highway Maintenance Equipment, Chang'an University,
}

${ }^{2}$ Key Laboratory of Road Construction Technology and Equipment of MOE, Chang'an University,
China ashibinchen520@sina.com, b1532207797@qq.com, c857699072@qq.com

*Corresponding author: Shi-Bin CHEN shibinchen520@sina.com

Keywords: Stereo garage, Supporting frame, Cylinder, Maximum stress, Maximum deformation.

\begin{abstract}
In order to guarantee structural safety of stereo garage and control its cost of construction, the key components, such as supporting frame and cylinder, were optimized by the ANSYS. Based on the static analysis of ANSYS, the results show that the maximum deformation of supporting frame is mainly distributed in the connection parts of axle and base. And the maximum stress is mainly concentrated in the connection parts of the cylindrical elements or cylindrical elements and truss. By removing redundant parts and employing the method of all-welled, the results show that the weight of the supporting frame is reduced from $117360 \mathrm{~kg}$ to $91065 \mathrm{~kg}$, which greatly saves the cost of production. And the stress is reduced from the original $7.9 \mathrm{MPa}$ to $4.8 \mathrm{MPa}$ in the cylinders. These provide an important basis for the structural safety and cost saving of the garage.
\end{abstract}

\section{Introduction}

With the vigorous development of China's national economy and the automobile industry, the number of private cars is increasing, so that the pressure of traffic is increasing rapidly in our country. At the same time, the parking problem has become more and more serious, especially in some high-tech zones and commercial areas, vehicles will not be parked for a long time, but it is hard to find a parking space $[1,2]$. Today, parking spaces in the number and layout has been far unable to meet the needs of reality, let alone meet the needs of modern urban development. How to transform and add parking spaces has become a common concern of all sectors of the community.

In recent years, stereo garage have many advantages, such as small floor area, high degree of automation, large storage capacity [3,4]. These cause the wide attention of scholars. Zhuoran Zhang designed the 6 layers stereo garage based on PLC, which mainly consisted of the entire structure, running procedure and electrical system design including hardware and program design ideas for lifting-traversing garage [5]. Lijie Wang investigated the basses of the intelligent measurement and control system used in underground stereo garage at the downtown business district, which has an important significance for popularization and application of stereo garage [6]. However, few scholars focus on the analysis and optimized design of the structure of the circular garage. Ruijun Zhang et al. innovatively designed the model of vertical rotary stereo garage based on TRIZ and AD, however they did not carry out a more detailed analysis and optimized design for the structure of the garage $[7,8,9]$.

In this paper, we propose a mechanical rotary stereo garage based on a gravity balance and introduce its overall structure. Next, the strength analysis and optimized design of the key parts are carried out by using ANSYS software under the action of self-weight, wind load and inertia load. 


\section{The Overall Structure and Static Analysis of Mechanical Rotary Stereo Garage \\ The Overall Structure of Stereo Garage}

Mechanical rotary stereo garage consists of the import and export module, access module, vehicle parking module and the wheel structure, as shown in Figure 1. The access of vehicles is completed by sliding way, as shown in Figure 2. And vehicles parked in the garage are on the form of active parking, as shown in Figure 3. From the perspective of the overall structure of stereo garage, the supporting frame plays a supporting role in the whole structure and the wheel is used for parking a single car. Both are key components of the garage, and their security and reliability are the key to ensure the smooth operation and safety of parking garage.

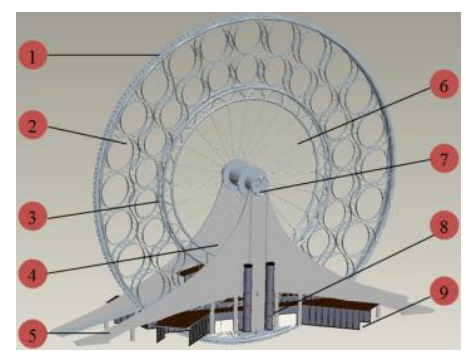

Figure 1. The overall structure of stereo garage

1-The chain of the outer ring of the wheel; 2-Cylindrical element; 3-The truss of wheel; 4-Supporting frame; 5-Access channel at second floor; 6-Rope; 7-Principal axis; 8-Elevator; 9-Access channel at first floor

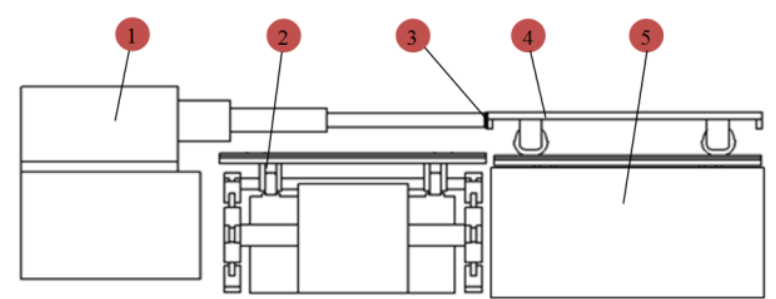

Figure 2. The working process of electromotive handspike

1-The electromotive handspike; 2-Alignment Vehicle platform; 3-Electromagnet; 4-Board of carrying car; 5-Cylindrical element

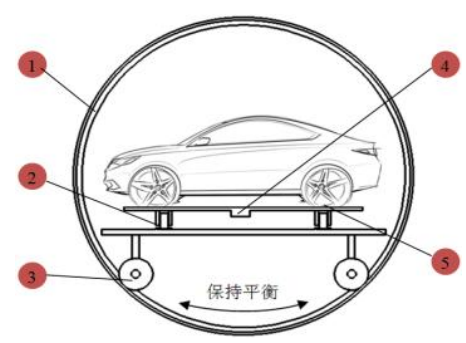

Figure 3. The structure of cylindrical element

1-Circular orbit; 2-The orbit groove of car carrying board; 3-The balance wheel; 4-The bulge of board of carrying car; 5-Antiskid equipment

\section{The Theoretical Analysis of Force of Key Components}

Stereo garage is a large component. The number of cylinder is 47 in the inner and outer layer. Due to the effective windward area of single cylinder unit is $7.2 \mathrm{~m}^{2}$, the total windward area is $7.2 \times 47=338 \mathrm{~m}^{2}$. And the maximum height of wheel can reach $80 \mathrm{~m}$. Therefore, in a static state, the supporting frame endures the gravity mostly, while the large wheel acts on the bearing bracket. But the wind load will have a huge impact on it from the dynamic perspective. The near-ground wind is 
relatively disordered, and the interference of wind is different at different height. In order to facilitate and simplify the calculation, the wind load on stereo garage is divided into 3 stable regions at $30 \mathrm{~m}, 40 \mathrm{~m}$ and $50 \mathrm{~m}$ according to the structural height.

Then, we employ the formula of standard value of wind load of the steel to calculate the wind load [10]:

$$
W_{k}=\beta_{z} \mu_{s} \mu_{z} W_{0}
$$

$W_{k}$ - standard value of wind load;

$\beta_{z}$ - wind-induced vibration coefficient at high Z;

$\mu_{s^{-}}$shape coefficient of wind load;

$W_{0}$ - the pressure of basic wind;

$\mu_{z}$ - the coefficient of height variation to wind load;

In this paper, we employ the 8 wind as the load condition of stereo garage $\left(W_{0}=51.5, \beta_{z}=16.5\right)$. According to the code for load of steel structure, we can know $\mu_{s}=0.55$, and when $\mathrm{z}=30 \mathrm{~m}, \mu_{z}=1.1$; when $\mathrm{z}=40 \mathrm{~m}, \mu_{z}=1.24$; when $\mathrm{z}=50 \mathrm{~m}, \mu_{z}=1.36$. Therefore, through the calculation of Eq.1, the maximum pressure of wind in each area is $515 \mathrm{~N} / \mathrm{m}^{2}, 580 \mathrm{~N} / \mathrm{m}^{2}$ and $636 \mathrm{~N} / \mathrm{m}^{2}$ respectively. In order to simplify the analysis, the equivalent wind load is two directions, that is, the wind load perpendicular and parallel to the end face of the garage.

\section{The Static Analysis of the Supporting Frame and the Wheel}

First, we apply Pro-E software to build 3D model of stereo garage, as shown in Figure 4, in which the number of inside and outside cylinder is 47 . Then, the 3D model is introduced into ANSYS, and the mesh is divided by free mesh, as shown in Figure 5. Finally, the load of the force and the solution are carried out, which assumes that the wind load is the 8 wind. The quality of wheel is $150 \mathrm{t}$, and the vehicle weighs $1.4 \mathrm{t}$ per car in outer layer, and the vehicle weighs $1.6 \mathrm{t}$ per car in inner layer, and the effective upwind area of a single cylindrical element is $7.2 \mathrm{~m}^{2}$ and the total windward area is $7.2 \times 47=338 \mathrm{~m}^{2}$. Considering the force of stereo garage according to the gradient wind, because the maximum pressure of the wind is respectively $515 \mathrm{~N} / \mathrm{m}^{2}, 580 \mathrm{~N} / \mathrm{m}^{2}$, $636 \mathrm{~N} / \mathrm{m}^{2}$ in each area, the wind load is determined to be $15000 \mathrm{~N} 、 4100 \mathrm{~N} 、 5700 \mathrm{~N}$ respectively, which are applied to the corresponding position of the wheel. And the wind load is perpendicular to the end face of the wheel. The stress cloud is shown in Figure 6, and Table 1 is the material properties.

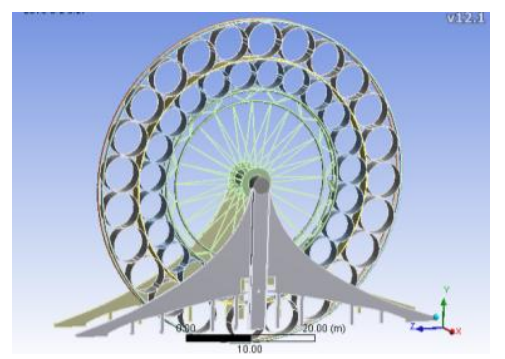

Figure 4. 3D model of stereo garage

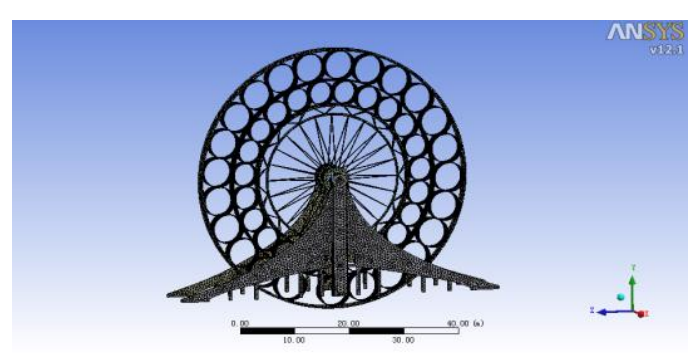

Figure 5. The Finite Element Meshing of Supporting frame

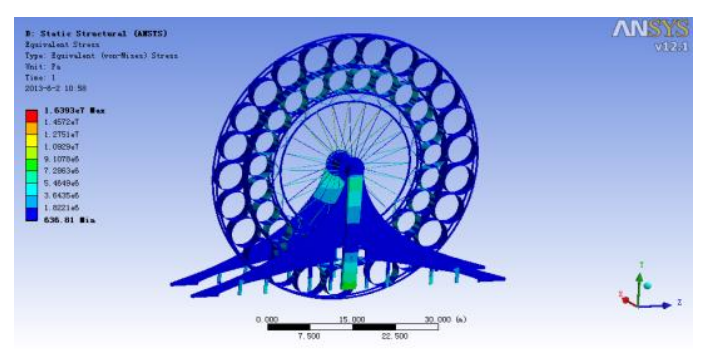

Figure 6. The stress analysis results of stereo garage 
Table 1. The material properties.

\begin{tabular}{|l|l|l|l|l|}
\hline Parameter & Elastic modulus $(\mathrm{Pa})$ & Density $\left(\mathrm{g} / \mathrm{m}^{3}\right)$ & Poisson's ratio & Yield strength $(\mathrm{MPa})$ \\
\hline Size & $2 \times 10^{11}$ & 7800 & 0.3 & 235 \\
\hline
\end{tabular}

The analysis results show that the total deformation of supporting frame is $6.5 \mathrm{~mm}$, and the deformation is mainly distributed in the connection part of axle and base, and the maximum stress is $9.1 \mathrm{MPa}$, which can meet the allowable value $(160 \mathrm{MPa})$ of structural steel. The total deformation of the wheel is $9.6 \mathrm{~mm}$, which is mainly concentrated in cylindrical elements of outer layer. The maximum stress of cylindrical elements is $16.7 \mathrm{MPa}$, which is mainly concentrated in the connection parts of the cylindrical elements and the connection parts of cylindrical elements and truss. In order to reduce the total deformation and the maximum stress of the wheel, it is necessary to further optimize the welding process between the cylindrical elements.

\section{The Optimized Design of Key Components of Mechanical Rotary Stereo Garage}

Through the static analysis, it is known that the maximum deformation of supporting frame is mainly no stable structure above it, so 4 stable cables are added above supporting frame. And due to the stress distribution of the supporting frame is relatively concentrated, the optimized design module of ANSYS software is used to optimize its structure. At the same time, the welding process of cylindrical elements is also optimized.

\section{The Optimized Design of Supporting Frame}

Under the condition of meeting the structural requirements, the whole quality of supporting frame should be reduced as much as possible. We take half load G1=200t and full load G2=243t respectively, and the analysis results are shown in Figure 7 and Figure 8. Red area represents removable part. Figure 9 is the scheme of the lightweight supporting frame with perfect appearance.

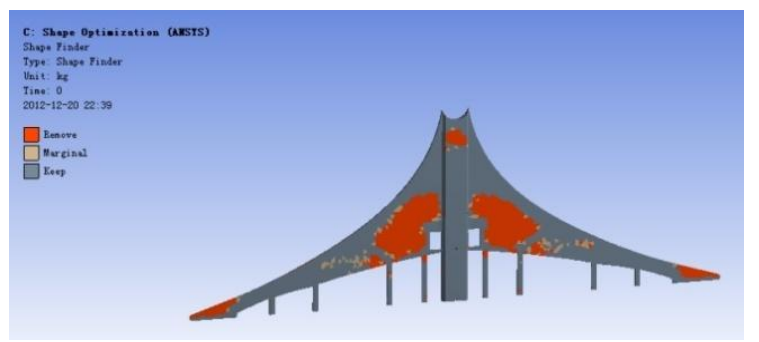

Figure 7. The stress distribution of supporting frame in the half load

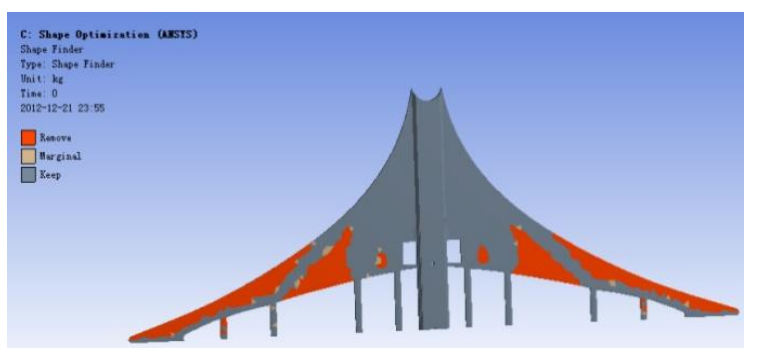

Figure 8. The stress distribution of supporting frame in the full load

Under the same loading condition and constraints, the finite element analysis of supporting frame shown in Figure 9 is carried. And the results are shown in Figure 10. The structural parameters of supporting frame are compared in the Table 2 before and after optimization. 


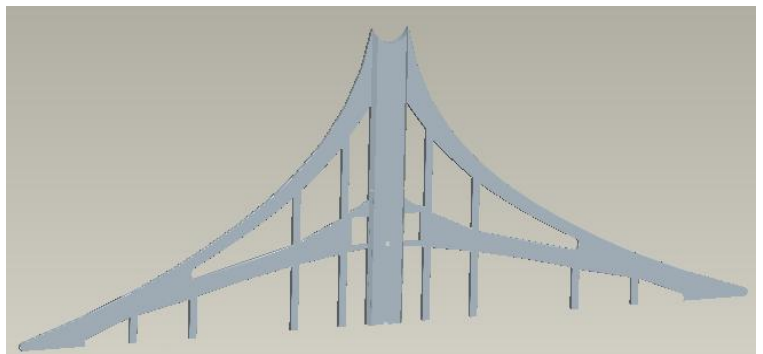

Figure 9. The optimized model of supporting frame

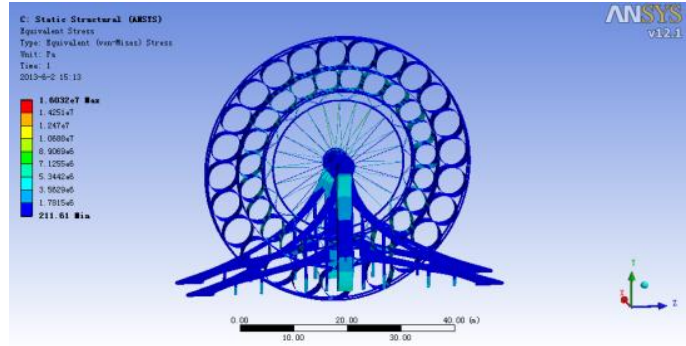

Figure 10. The stress distribution of optimized supporting frame

Table 2. Comparison of structural parameters of the support frame before and after optimization.

\begin{tabular}{|l|l|l|}
\hline Parameters & Before optimization & After optimization \\
\hline Maximum (mm) & 6.5 & 7.08 \\
\hline maximum stress (Mpa) & 9.1 & 8.9 \\
\hline Quality (kg) & 117360 & 91065 \\
\hline
\end{tabular}

As can be seen from Table 2, the maximum deformation and the maximum stress have little change on the original basis, while the weight is reduced $22.4 \%$, which greatly reduces the cost of production.

\section{The Optimized Design of Cylindrical Elements}

In this paper, we employ the orthogonal experimental method to optimize layout on the cylindrical elements. Through a lot of experiments we can know:

Under the same conditions, different welding points have large difference on the influence of maximum stress. For instance, when the six points are welded at the connection of three units, namely all-welled, the maximum stress and maximum deformation of cylindrical elements are both minimum. But it will choose four welding points in three cylindrical elements under more difficult circumstance which calls all-welled.

Aimed at the design scheme of the mechanical rotary stereo garage, we employ the program that all contacted surfaces are welded, namely the six points are welded at connection of three units, and the loading distribution is shown at Figure 11. Cylindrical elements of the internal and external are endured respectively $1.4 \mathrm{t}$ and 1.6t, as well as wind pressure on the wheel at the different region and weight of wheel are acted. Now the analysis results are shown on Figure 12 and Figure 13 separately before optimization and behind optimization.

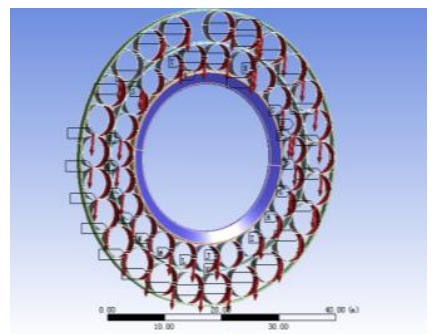

Figure 11. The load distribution of the wheel 


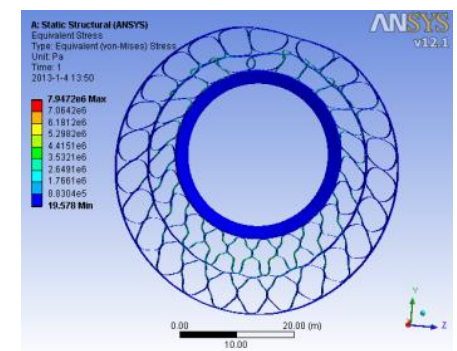

Figure 12. The stress distribution of the layout and solder joint before optimization

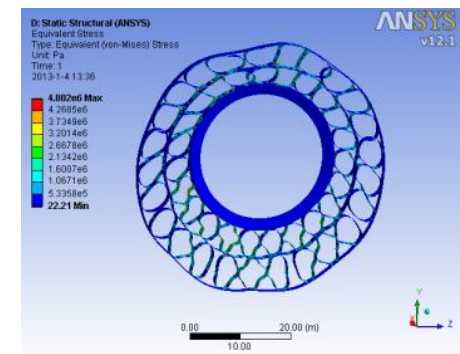

Figure 13. The stress distribution of the layout and solder joint after optimization

From the result of analysis we can see that the maximum stress of the wheel is reduced from the original $7.9 \mathrm{MPa}$ to $4.8 \mathrm{MPa}$, nearly half of the strain degree is reduced by employing the method of six points welded at the connection of three elements. And the maximum deformation is improved obviously.

\section{Conclusions}

By the force analysis to stereo garage, the results show that the wind has great impact on it. Then the ANSYS software is used to optimize the supporting frame and cylinder elements.

(1) The results of static analysis to stereo garage show that maximum deformation of the supporting frame is mainly distributed in the connection part of axle and base. And 4 stable cables are added at the top of it to enhance its stiffness. Then, we optimize the structure of the supporting frame and find that its weight is reduced from $117360 \mathrm{~kg}$ to $91065 \mathrm{~kg}$ under the little deformation and stress variation, which greatly reduce the cost of production.

(2) The results of static analysis to wheel show that the stress is mainly concentrated in the connection parts of the cylindrical elements or cylindrical elements and truss. Then, the way of six points welded at the connection of three elements is carried out, which shows that the maximum stress of the wheel is reduced from the original 7.9MPa to $4.8 \mathrm{MPa}$, nearly reducing half of degree. And the maximum deformation is improved obviously. These provide a basis for the safety and cost saving of the garage.

\section{Acknowledgements}

This work is supported by National Natural Science Foundation of China (No. 51508031), China Postdoctoral Science Foundation (No.2015M582583) and Fundamental Research Funds for the Central Universities of China (No. 310825163408 and 310825153403 ).

\section{References}

1. Manli Cha, Mingxing Lin, Monitoring system for stereo garage, Chinese Journal of scientific instrument 27,6 (2006)

2. Mingsheng Qu, Sun Wei, Deqiang Jing, Car storage-retrieval safety problems in up-and-down translation parking equipment, Hoisting and Conveying Machinery 10 (2012) 
3. Yubin Zhang, Yunping Yao, Zhengang Wu, Intelligent Infusion Device Control System Based on Chip Microcomputer. International Forum on Information Technology and Applications (2010)

4. Holton, Amy E., Fisher, Donald L, Advanced parking management systems: Models of drivers' Parking strategies, Proeeedings of the Human Factors and Ergonomies Society 3 (2003)

5. Zhuoran Zhang, Zhe Mao, Hailing Wan, THE 6 LAYERS STEREO GARAGE DESIGN BASED ON OMRON PLC. International Conference on Electronic \& Mechanical Engineering and Information Technology (2011)

6. Lijie Wang, Jing $\mathrm{Xu}$, Hongyu Sun, Design and analysis of parking guidance system for underground intelligent stereo garage in downtown business district, International Conference on Instrumentation, Measurement, Computer, Communication and Control (2013)

7. Ruijun Zhang, Mingqin Zhang, Xiaowei Wang, Lifang Han, Min Li, The innovative design on the scheme of the vertical turn stereo garage based on TRIZ and AD, Technology and Innovation Conference 1, 6 (2009)

8. D.-K. Kim and B.-I. Kwon, A Novel Equivalent Circuit Model of Linear Induction Motor based on Finite Element Analysis and its Coupling with External Circuits, IEEE Trans. Magn 42, 5 (2006)

9. Q.F. Lu, Y.X. Li, Y.Y. Ye, Z.Q. Zhu, Investigation of Forces in Linear Induction Motor Under Different Slip Frequency for Low-Speed Maglev Application, IEEE Trans. Energy Convers 28, 1 (2013)

10. Thermal Loading and Reliability of 10-MW Multilevel Wind Power Converter at Different Wind Roughness Classes, IEEE Energy Conversion Congress and Exposition (ECCE) (2012) 